\title{
Ligand binding and conformational changes of SUR1 subunit in pancreatic ATP-sensitive potassium channels
}

\author{
Jing-Xiang $\mathrm{Wu}^{1,2}$, Dian Ding ${ }^{1,2,3}$, Mengmeng Wang ${ }^{1,2,3}$, Yunlu Kang ${ }^{1,2}$, Xin Zeng $^{1,2,3}$, Lei Chen ${ }^{1,2 \bowtie}$ \\ ${ }^{1}$ State Key Laboratory of Membrane Biology, Institute of Molecular Medicine, Peking University, Beijing Key Laboratory of \\ Cardiometabolic Molecular Medicine, Beijing 100871, China \\ 2 Peking-Tsinghua Center for Life Sciences, Peking University, Beijing 100871, China \\ ${ }^{3}$ Academy for Advanced Interdisciplinary Studies, Peking University, Beijing 100871, China \\ $\bowtie$ Correspondence: chenlei2016@pku.edu.cn (L. Chen)
}

Received March 6, 2018 Accepted March 14, 2018

\begin{abstract}
ATP-sensitive potassium channels ( $\left.\mathrm{K}_{\mathrm{ATP}}\right)$ are energy sensors on the plasma membrane. By sensing the intracellular ADPIATP ratio of $\beta$-cells, pancreatic $K_{\text {ATP }}$ channels control insulin release and regulate metabolism at the whole body level. They are implicated in many metabolic disorders and diseases and are therefore important drug targets. Here, we present three structures of pancreatic $\mathrm{K}_{\text {ATP }}$ channels solved by cryoelectron microscopy (cryo-EM), at resolutions ranging from 4.1 to $4.5 \AA$. These structures depict the binding site of the antidiabetic drug glibenclamide, indicate how Kir6.2 (inward-rectifying potassium channel 6.2) N-terminus participates in the coupling between the peripheral SUR1 (sulfonylurea receptor 1) subunit and the central Kir6.2 channel, reveal the binding mode of activating nucleotides, and suggest the mechanism of how Mg-ADP binding on nucleotide binding domains (NBDs) drives a conformational change of the SUR1 subunit.
\end{abstract}

KEYWORDS $\mathrm{K}_{\mathrm{ATP}}$, SUR, ABC transporter, glibenclamide, sulfonylurea, diabetes

\section{INTRODUCTION}

ATP-sensitive potassium channels $\left(\mathrm{K}_{\mathrm{ATP}}\right)$ are ion channels that selectively allow potassium ions to permeate the cell.

Electronic supplementary material The online version of this article (https://doi.org/10.1007/s13238-018-0530-y) contains supplementary material, which is available to authorized users.
Their channel activities are tightly regulated by endogenous nucleotide metabolites. Specifically, they are inhibited by ATP and activated by Mg-ADP. By sensing the intracellular ADP/ATP ratio, $K_{\text {ATP }}$ channels tune the potassium ion efflux across the plasma membrane and adjust the membrane potential. Therefore, $\mathrm{K}_{\text {ATP }}$ channels convert the cellular metabolic status into electrical signals, a unique output that has broad physiological effects. $\mathrm{K}_{\text {ATP }}$ channels are widely distributed in many tissues, including the pancreas, brain, heart, and smooth muscle (Hibino et al., 2010), and they play important roles in many physiological processes, such as hormone secretion and vasodilatation (Hibino et al., 2010). Genetic mutation of genes that encode $\mathrm{K}_{\text {ATP }}$ channel subunits can lead to several metabolic diseases and neuronal diseases (Ashcroft et al., 2017; Hibino et al., 2010). Therefore, $\mathrm{K}_{\text {ATP }}$ channels are important drug targets. Clinically relevant sulfonylureas drugs inhibit pancreatic $\mathrm{K}_{\text {ATP }}$ channels and serve as insulin secretagogues for the treatment of type II diabetes (Bryan et al., 2005), while $\mathrm{K}_{\text {ATP }}$ activators, such as potassium channel openers (KCOs), are used for hypoglycemia and show promise for myoprotection (Flagg et al., 2010; Hibino et al., 2010).

Previous studies have established that the functional $\mathrm{K}_{\text {ATP }}$ channel is a hetero-octamer composed of four inwardrectifying potassium channel 6 (Kir6) subunits and four sulfonylurea receptor (SUR) regulatory subunits (Clement et al., 1997; Shyng and Nichols, 1997; Ueda et al., 1997). Kir6 subunits are encoded by either KCNJ8 (Kir6.1) or KCNJ11 (Kir6.2). Kir6 subunits harbor sites for inhibitory ATP binding. The activities of Kir6 can be enhanced by $\mathrm{PIP}_{2}$, which is a signaling lipid present in the inner leaflets of the plasma membrane. SUR subunits are composed of the N-terminal 
transmembrane domain 0-loop 0 (TMD0-L0) and ATP-binding cassettes $(A B C)$ transporter-like modules. They are encoded by either $A B C C 8$ (SUR1) or ABCC9 (SUR2). SUR subunits bind stimulatory Mg-ADP, KCOs, and inhibitory sulfonylureas and regulate Kir6 channel activity. $\mathrm{K}_{\mathrm{ATP}}$ channels composed of different isoform combinations are distributed in different tissues and have distinct pharmacological profiles, which are primarily determined by the identity of associated SUR subunits.

The $A B C$ transporter has two nucleotide binding domains (NBDs). Each NBD harbors two adjacent halves of a catalytic site for ATP hydrolysis. Therefore, one NBD must pair with the other NBD to form two functional ATP hydrolysis sites when they are in proximity (Locher, 2016). SUR proteins belong to subfamily $C$ of the $A B C$ family transporters $(A B C C)$. The other two family members of $A B C C$ are multidrug resistance-associated proteins (MRPs) and the cystic fibrosis transmembrane conductance regulator (CFTR). MRP is a transporter with known substrates, and the transporter activity of MRP depends on the hydrolysis of Mg-ATP, whereas the CFTR is an Mg-ATP-gated chloride channel. The hallmark of the $A B C C$ family is that the conserved glutamate residue on NBD1 Walker $B$ motif of canonical $A B C$ transporters is replaced by an aspartate (D855 in SUR1). Therefore, the catalytic activity of one ATP hydrolysis site (degenerate site or nucleotide binding site 1, NBS1) is lost (Aittoniemi et al., 2009), and only one functional ATP hydrolysis site (consensus site or NBS2) remains. The activities of most $A B C$ transporters depend on the hydrolysis of Mg-ATP. However, Mg-ADP is the primary nucleotide species that activates SUR1 to enhance $\mathrm{K}_{\text {ATP }}$ channel activity (Aittoniemi et al., 2009).

Because of the physiological importance of pancreatic $\mathrm{K}_{\mathrm{ATP}}$ channels, they have served as a model to study the biochemical and physiological properties of $\mathrm{K}_{\text {ATP }}$ channels for decades. $\mathrm{K}_{\text {ATP }}$ channels in pancreatic islets are composed of Kir6.2 channel subunits and SUR1 regulatory subunits. Recently, several structures of pancreatic $K_{\text {ATP }}$ channel are solved by us and other groups, including the GBM bound inhibited state (abbreviated, GBM state, EMD6689) (Li et al., 2017), the ATP + GBM bound inhibited state (abbreviated, ATP + GBM state, EMD-7073 and EMD-8470) (Martin et al., 2017a; Martin et al., 2017b) and the Mg-ATP and Mg-ADP bound state (abbreviated, Mg-ATP\&ADP state, EMD-7338 and EMD-7339) (Lee et al., 2017). These structures reveal the architecture of $\mathrm{K}_{\text {ATP }}$ channels, the details of how different subunits are assembled together, the binding site of sulfonylureas on SUR1, ATP on Kir6.2, and Mg-ADP/ ATP on SUR1. However, the mechanisms of the sulfonylureas inhibition and Mg-ADP activation are still lacking.

In this paper, we present three structures of the pancreatic $\mathrm{K}_{\text {ATP }}$ channel solved using an SUR1-Kir6.2 fusion construct. The first structure is the $\mathrm{K}_{\text {ATP }}$ channel in complex with ATPYS and GBM, and it is solved to a resolution of $4.3 \AA$ (abbreviated, ATP + GBM state). The second structure is the $\mathrm{K}_{\mathrm{ATP}}$ channel in complex with ATPYS alone, which is solved to $4.4 \AA$ resolution (abbreviated, ATP state). The third structure is the $\mathrm{K}_{\text {ATP }}$ channel in complex with Mg-ADP, $\mathrm{VO}_{4}{ }^{3-}, \mathrm{PIP}_{2}$ and NN414 (Carr et al., 2003), a ligand combination that favors the NBD-dimerized state of SUR1, which is solved to $4.3 \AA$ (abbreviated, Mg-ADP state). This new structural information, together with previously solved structures, provides mechanistic insights into how the pancreatic $\mathrm{K}_{\text {ATP }}$ channel works.

\section{RESULTS AND DISCUSSION}

\section{A $\mathrm{K}_{\text {ATP }}$ channel fusion construct for cryo-EM structure determination}

To overcome the compositional heterogeneity of the $\mathrm{K}_{\mathrm{ATP}}$ channel when heterologously co-expressing SUR1 with Kir6.2 (Li et al., 2017), we created a SUR1-Kir6.2 fusion construct, in which the intracellular C-terminus of SUR1 is covalently linked to the N-terminus of Kir6.2 by a linker (Fig. S1A). Fusion construct with a 6 amino acids (6AA) linker was previously used to elucidate the association and stoichiometry of K $\mathrm{K}_{\text {ATP }}$ channels (Clement et al., 1997) and was recently used to solve the structure of $\mathrm{K}_{\text {ATP }}$ channel in complex with Mg-ATP and Mg-ADP (Lee et al., 2017). To avoid potential artifacts on the structure of the $\mathrm{K}_{\text {ATP }}$ channel because of limited linker length, we used a flexible linker with 39 amino acids (39AA), which should be sufficient for a $130 \AA$ linear distance when fully extended. Similar to the wild-type channel, the fusion construct can be inhibited by ATP and activated by Mg-ADP in an inside-out patch clamp, which is the characteristic property of $\mathrm{K}_{\text {ATP }}$ channel (Fig. S1B). However, inhibition by GBM is markedly decreased (Fig. S1B). Because it has been reported that the individually expressed SUR1 subunit is sufficient for high affinity GBM binding (Aguilar-Bryan et al., 1995) and our construct has an intact SUR1 subunit, we reasoned that the reduced GBM inhibition is due to insufficient coupling between SUR1 and Kir6.2 of our fusion construct, as discussed later. To be noted, it is reported that $1 \mu \mathrm{mol} / \mathrm{L}$ GBM can fully inhibit the 6AA linker construct in whole cell ${ }^{86} \mathrm{Rb}^{+}$efflux assays (Shyng and Nichols, 1997), this is probably because the GBM can bind SUR1 to counteract the activation effect of intracellular Mg-ADP or Mg-ATP, rather than directly inhibits the 6AA fusion channel. It is also reported that $300 \mu \mathrm{mol} / \mathrm{L}$ tolbutamide can attenuate the currents of $6 \mathrm{AA}$ construct in inside-out patch clamp recording (Lee et al., 2017). This is possibly due to the low affinity block of sulfonylurea on Kir6.2 channel (Reimann 1999). Whether the 6AA construct can be directly inhibited by the GBM bound on SUR1 remains to be thoroughly determined.

\section{Structures of the $\mathrm{K}_{\text {ATP }}$ channel in the ATP + GBM and ATP states}

We aimed to obtain structures of $\mathrm{K}_{\text {ATP }}$ with and without GBM at higher resolution than our previous GBM state (Li et al., 2017), which can be used to locate the GBM density by 

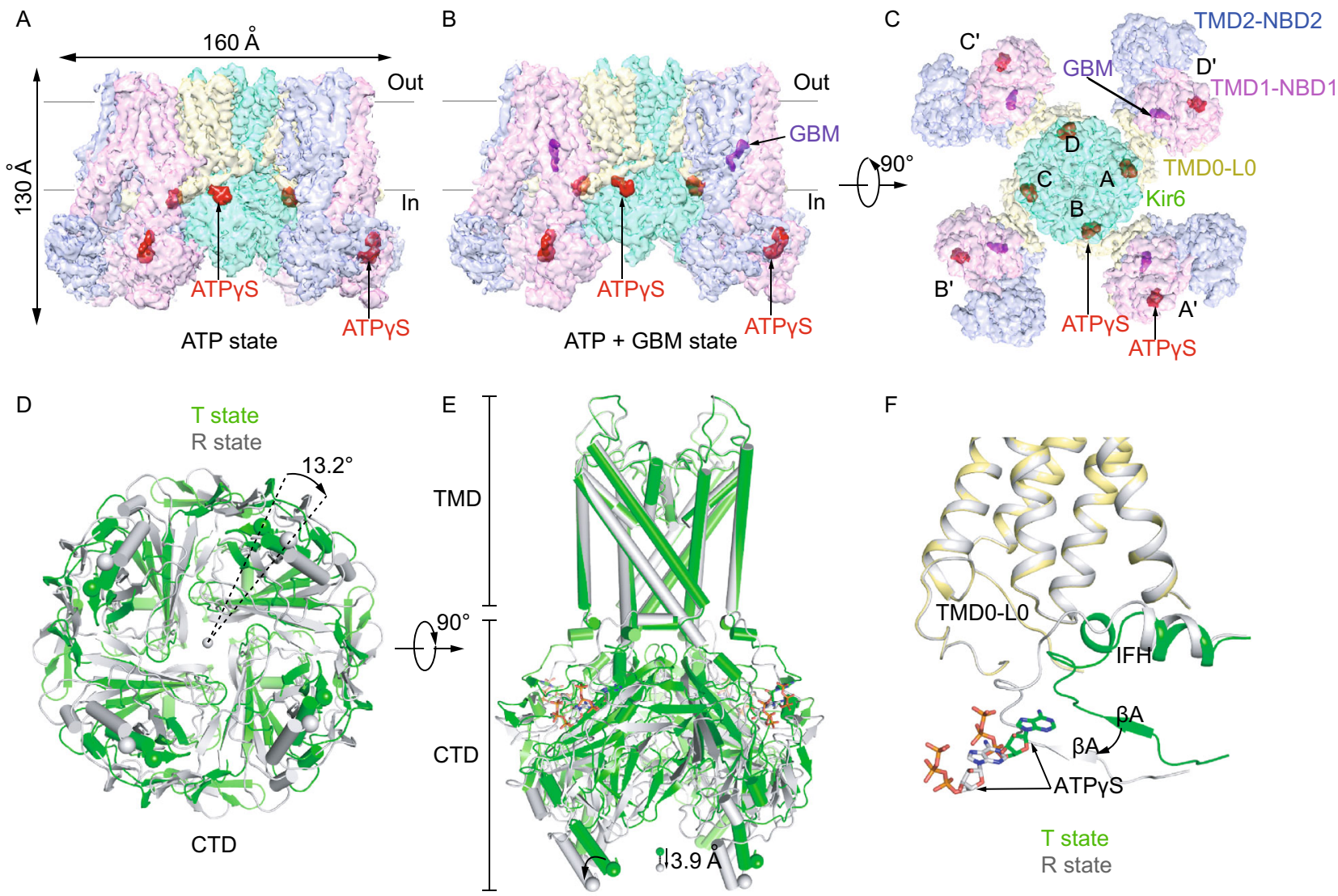

$\mathrm{F}$

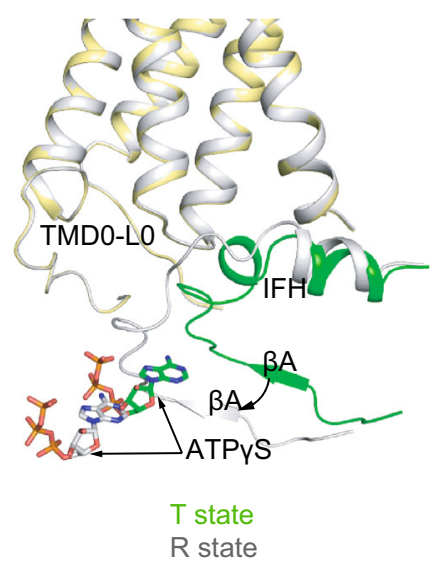

Figure 1. Structures of the pancreatic $K_{A T P}$ channel in complex with ATPYS or ATPYS and glibenclamide (GBM). (A) Side view of the $\mathrm{K}_{\text {ATP }}$ channel in complex with ATPYS (ATP state). Approximate extent of the phospholipid bilayer is shown as thick gray lines. Kir6.2, SUR1 TMD0-L0 (transmembrane domain 0-loop 0) fragment, TMD1-NBD1 (nucleotide binding domain1), TMD2-NBD2, ATPYS and GBM are shown in green, yellow, magenta, blue, red and purple, respectively. (B) Side view of the $\mathrm{K}_{\text {ATP }}$ channel in complex with ATPYS and GBM (ATP + GBM state), colored the same as in (A). (C) Bottom view of the $\mathrm{K}_{\text {ATP }}$ channel in complex with ATPYS and GBM from the intracellular side. (D) Bottom view of the aligned Kir6.2 structures between "T state" (green) and "R state" (gray) of the ATP + GBM state. Rotation angle between CTDs (cytoplasmic domain) was measured using Ca positions of L356 of Kir6.2, shown as spheres. (E) Side view of the aligned Kir6.2 structures between "T state" (green) and "R state" (gray) of the ATP + GBM state. Vertical movement of CTDs is measured between the centers of mass of L356 Cas. (F) Positional difference of Kir6. $2 \beta A$ and IFH (interfacial helix) between "T state" and "R state" structures of the ATP + GBM state.

direct comparison. Our previous study demonstrated that the $\mathrm{K}_{\text {ATP }}$ channel displays a large degree of conformational heterogeneity when in complex with the high affinity inhibitor GBM alone (Li et al., 2017). The additional ATP molecule, an endogenous inhibitor of $\mathrm{K}_{\text {ATP }}$, can further stabilize the Kir6.2 subunit to reduce conformational heterogeneity (Martin et al., 2017b). Therefore, we applied ATPYS, a slowly hydrolyzable and functional ATP analog, to stabilize the structures of the $\mathrm{K}_{\text {ATP }}$ channel with and without GBM (Schwanstecher et al., 1994a).

The fusion channel protein was purified and then subjected to cryo-EM single particle analysis (Fig. S1C and S1D). The structure of $K_{\text {ATP }}$ in complex with ATPYS and GBM was solved at an average resolution of $4.3 \AA$, slightly higher than the structure in complex with ATPYS alone at a resolution of $4.5 \AA$ (Figs. $1 \mathrm{~A}-\mathrm{C}, \mathrm{S} 2, \mathrm{~S} 3 \mathrm{~A}$, and Tables S1 and
S2). The overall $K_{\text {ATP }}$ channel structure and SUR1 conformation in the ATP + GBM state is almost identical to that in the ATP state based on our density maps (Fig. S3B). Therefore, we focus the discussion on the structure of the ATP + GBM state with a higher resolution, unless stated otherwise.

Focused three-dimensional (3D) classification on the Kir6.2 cytosolic globular domain (CTD) isolated two major classes with distinct conformations (Fig. S2 and Table S1). The CTDs of one class have relative rotation to the other, as observed previously in the structure of the ATP + GBM state (Martin et al., 2017b). Each class was individually refined to reach resolutions of $4.3 \AA$ or $4.6 \AA$. In contrast, the transmembrane domain (TMD) of the two classes shares the same structure and focused refinement with all particles reached an overall resolution of $4.1 \AA$ (Fig. S2C and 
Table S1). The maps demonstrate side chain densities for most residues and enable further improvement of our previous model based on medium resolution maps. The overall structures of both classes are similar to the previous symmetric Class 1.1 structure of the GBM state (EMD-6689) (Li et al., 2017) or that of the ATP + GBM state (EMD-8470) (Martin et al., 2017b), with the central Kir6.2 in a closed state and the peripheral SUR1 in an inward-facing inactive state (Fig. 1B and 1C). By comparing the structures of the two classes, we found that the CTDs of Kir6.2 (R32 to L66 and $\mathrm{K} 170$ to L356) move in a rigid body fashion relative to the transmembrane domains, resulting in a $3.9-\AA$ downward movement and $13.2^{\circ}$ clockwise rotation (Fig. 1D and 1E). In contrast, the structure of transmembrane domain of Kir6.2 and SUR1 is largely unchanged (Fig. 1D and 1E). Therefore, the interface between Kir6.2 CTD and SUR1 TMD0-L0 fragment is altered and the conformations of the linkers between the two rigid bodies also change. Here, we refer to class 1 structure as the "T state" (the tense state) and class 2 as the "R state" (the relaxed state). Specifically, in the " $R$ state" structure, the linker between $\beta A$ and the interfacial helix (IFH) of Kir6.2 moves away from the TMD0-L0 of SUR1 (Fig. 1F). The differences between the two conformations described herein are not due to differences in ATPYS occupancy on CTDs, because we observed similarly strong ATPYS densities in the maps of both classes (Fig. S4A). Instead, they possibly reflect the endogenous mobility of the CTDs in the Kir6.2-inhibited state, because we observed similar conformational heterogeneity of the $\mathrm{K}_{\text {ATP }}$ channel in the ATP state and the Mg-ADP state as described later. The co-existence of "T state" and "R state" might be functionally important since the rotation of CTD relates to channel gating in another Kir channel member GIRK2 (Whorton and MacKinnon, 2011, 2013) .

\section{GBM binding site on SUR1}

Previous studies demonstrated that mutations of $Y 230 A$ and W232A on the lasso motif reduce GBM affinity (Vila-Carriles et al., 2007), indicating the lasso motif might be in proximity to or on the binding site of GBM. Therefore, we compared the density maps around lasso motif between the ATP state and the ATP + GBM state, and found that the extra density near the lasso motif in the previous medium resolution map is present in both states. With improved resolution and map quality, we found that this extra density was contributed by both the M5-Lh1 loop and an unknown ligand, probably a lipid head group that constitutively binds $\mathrm{K}_{\mathrm{ATP}}$, because it is also present in the Mg-ADP state described later (Fig. S4B). Instead, a previously unmodeled density inside the SUR1 central cavity in the ATP + GBM state map has a distinct elongated shape that matches the GBM molecule in an extended conformation (Figs. 2A and S4C-E). In contrast, this density is not present in the ATP state, where GBM was not supplied into the sample (Fig. 2B). This unambiguously demonstrates that the extra density inside SUR1 central
Figure 2. Binding sites of ATPYS and GBM on SUR1. (A) Occupied GBM binding site in the $4.4 \AA$ ATP + GBM state map after focus refinement of SUR1 ABC transporter module. The density of GBM is shown as green mesh with GBM molecule shown in stick representation. The putative KNtp (Kir6.2 N-terminus) binding site is outlined by green dash lines. (B) Apo GBM binding site in the $4.4 \AA$ ATP state map. Maps in (A) and (B) are low-pass filtered to $4.5 \AA$ for comparison. (C-F) EM densities of putative KNtp. Extra continuous density close to GBM molecule is colored in green and GBM density is shown in purple. For better visualization of low resolution features, maps were sharpened with lower B factor than determined by postprocessing process. Maps of the GBM state (two half maps for EMD-6689 entry) (C), ATP + GBM state (EMD7073) (D and E), and ATP + GBM state with SUR1-Kir6.2 fusion construct $(F)$ were sharpened with $B$ factors -200 , -50 , and +200 , and contoured at $0.4,1.4$ and 0.015 levels, respectively. To illustrate the position of $\mathrm{KNtp}$ relative to the rest of the Kir6.2 channel, the side view of a single Kir6.2 and SUR1 subunit is shown in (E). (G) Sequence alignment of KNtp of Mus musculus Kir6.2 (mmKir6.2), Mus musculus Kir6.1 (mmKir6.1), Homo sapiens Kir6.2 (hsKir6.2), and Danio rerio Kir6.2 (drKir6.2). Because KNtp is unmodeled due to limited map quality, it is shown as dashed lines above. Nonconserved residues are highlighted in red. Position of the inserted dipeptide (GlySer) is indicated. $(\mathrm{H})$ Comparison of GBM inhibitory effects among Kir6.2 constructs with different numbers of Gly-Ser (GS) dipeptides inserted or N-terminal deletion, when coexpressed with SUR1. The inhibitory effect of GBM on SUR1-Kir6.2 fusion construct is also shown. Currents after GBM treatment were normalized to the currents before GBM treatment. Each bar represents mean \pm standard error, $n=3$. (I) EM densities at the degenerate site of SUR1 in the ATP + GBM state. Densities of ATPYS and surrounding region are shown as green and blue meshes, respectively. Figure is created from map after focused refinement of SUR1 ABC transporter module.

cavity in the map of the ATP + GBM state represents the GBM molecule (Figs. 2A and S4C-E). The extended conformation of GBM bound to SUR1 is dramatically different from the compact conformation of GBM bound to human aldo-keto reductase $1 \mathrm{C} 3$ subfamily (AKR1Cs) (Zhao et al., 2015) (Fig. S4C). Our results confirmed the GBM binding site reported by another group recently (PDB: 6BAA, EMD7073) (Martin et al., 2017a). Accompanied by mutagenesis results, they suggested indirect allosteric effects of Y230A or W232A. These mutations on the lasso motif might change the structure of adjacent transmembrane helices that are essential for GBM binding (Martin et al., 2017a). Therefore, mutagenesis results might be affected by allostery, and comparison between the maps of $\mathrm{K}_{\text {ATP }}$ solved from samples 

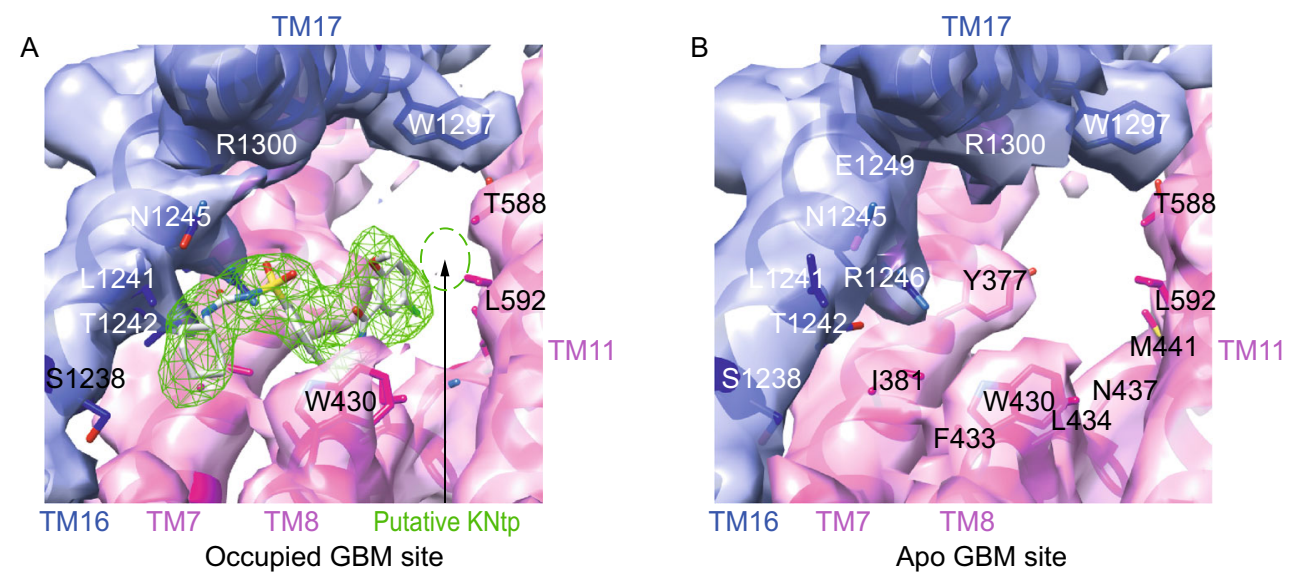

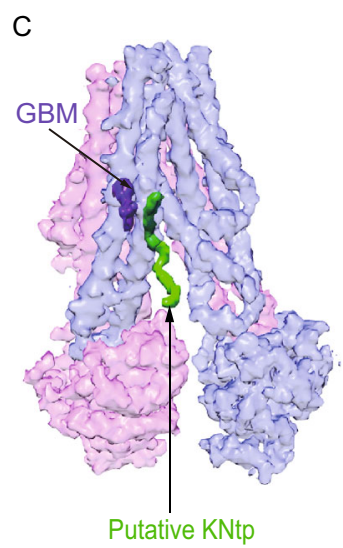

GBM state (EMD-6689) SUR1 + Kir6.2
D

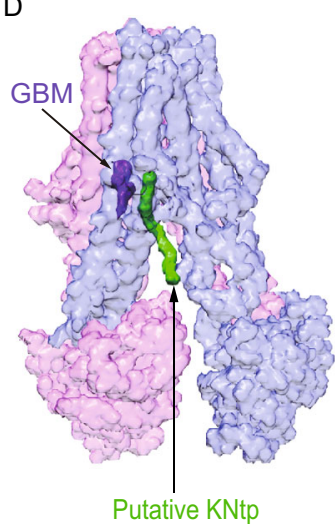

E

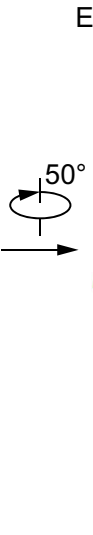

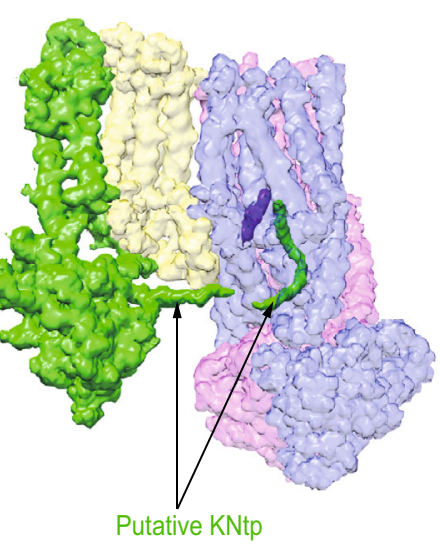

$\mathrm{F}$
G

mmKir6.2 1 MLSRKGI IPEEYVLTRL-AEDPAEPRYRTRERRARF 35 mmKir6.1 1 MLARKSI IPEEYVLARIAAENLRKPR I RDRLPKARF 36 hsKir6.2 1 MLSRKGI IPEEYVLTRL-AEDPAKPRYRARQRRARF 35 drKir6.2 1 MLSRKGLIPEDYLLTRL-AEDVLQPKFKTKQRKARF 35

$\mathrm{H}$

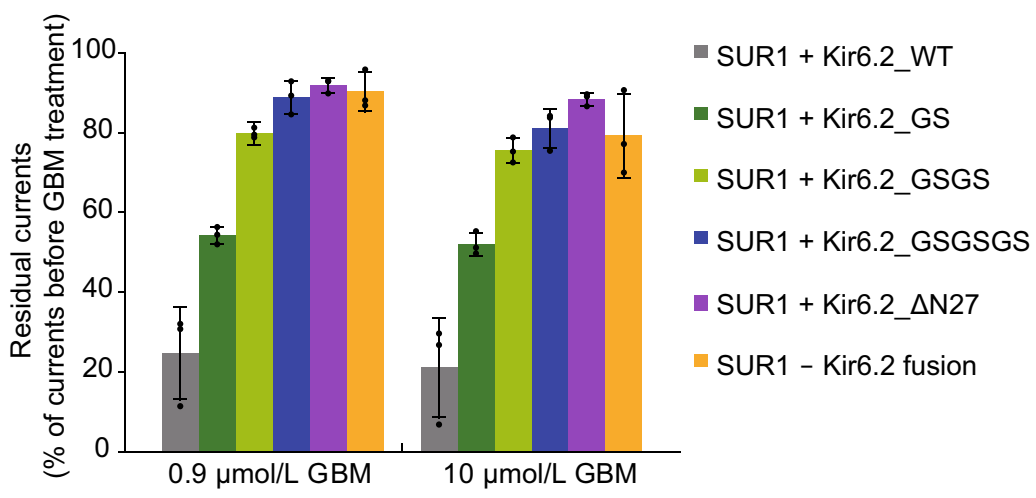

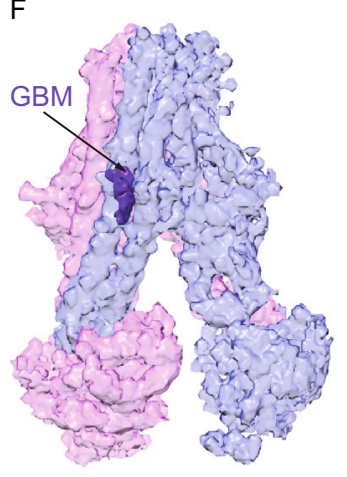

ATP + GBM state SUR1-Kir6.2 fusion

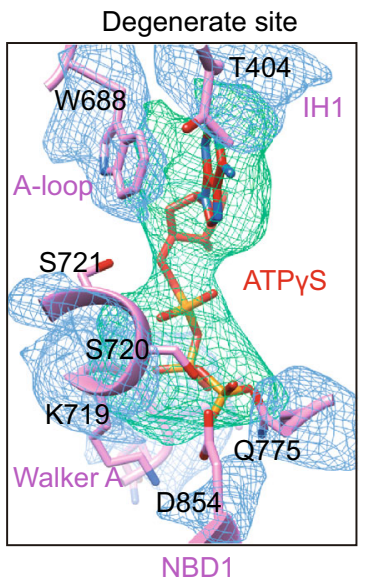


supplied with and without GBM is essential to definitively assign the non-protein density for GBM.

\section{Location of Kir6.2 N-terminus}

The fact that we observed strong GBM density inside SUR1 further reinforced our above assumption that SUR1-Kir6.2 fusion protein can bind GBM but the reduced GBM inhibition is because of insufficient coupling between SUR1 and Kir6.2 due to the covalent linker (Fig. S1B). When docking the improved model of SUR1 and GBM into our previous Class 1.1 map of the GBM state (EMD-6689) (Li et al., 2017), we found an additional positive density in the SUR1 central cavity that originates from the proximity of the GBM molecule and extends out of the SUR1 ABC transporter module in the direction of Kir6.2 CTD (Fig. 2C). Our previous GBM state structure (Li et al., 2017) and the ATP + GBM state structure by another group (Martin et al., 2017b) were solved using $\mathrm{K}_{\text {ATP }}$ protein generated by the coexpression of SUR1 with Kir6.2 as separated polypeptides; therefore, the Kir6.2 protein had a native $\mathrm{N}$-terminus in these studies. In the map of the ATP + GBM state (EMD-7073), we observed a similar density (Fig. 2D and 2E). Unfortunately, because of limited local map quality, we cannot infer the identity of this density purely from these maps. In contrast, we did not observe any density at the same position in our map of the ATP + GBM state that was solved using the SUR1-Kir6.2 fusion construct, in which the $\mathrm{C}$-terminus of SUR1 is covalently connected to the $\mathrm{N}$-terminus of Kir6.2 by a linker (Fig. 2F) and the linker together with Kir6.2 $\mathrm{N}$-terminus is completely disordered in our cryo-EM map. In our previous models (PDB: 5WUA) (Li et al., 2017), which were built according to the cryo-EM map, only one amino acids of the SUR1 C-terminus were unmodeled, and they are unlikely to contribute to such an elongated unaccounted density. Therefore, this positive density, which is close to the GBM binding site, might represent the $\mathrm{N}$-terminus peptide of Kir6.2 with 30 amino acids omitted in the model. This observation agrees with previous biochemical data showing that Kir6. 2 can be labeled by ${ }^{125} \mathrm{I}$-azidoglibenclamide and deletion of KNtp reduced not only ${ }^{125} \mathrm{I}$-azidoglibenclamide labeling (Babenko and Bryan, 2002; Vila-Carriles et al., 2007) but also the affinity for GBM and repaglinide (Kuhner et al., 2012). In addition, GBM enhanced the cross-linking of SUR1 with Kir6.2 with the photo cross-linkable amino acid incorporated in the $\mathrm{N}$-terminus (Devaraneni et al., 2015), indicating that the KNtp should be in proximity to SUR1. This is further supported by the electrophysiological data that high affinity inhibition of tolbutamide, GBM and repaglinide to the $\mathrm{K}_{\text {ATP }}$ channel were either abolished or markedly decreased when KNtp is deleted (Devaraneni et al., 2015; Kuhner et al., 2012; Reimann, 1999). Moreover, the density of the putative KNtp is closed to the 1-chloro-4-methoxybenzene group of GBM (Figs. 2A and $\mathrm{S} 4 \mathrm{E}$ ), the so called "B" site, where the azido group in ${ }^{125} \mathrm{I}-$ azidoglibenclamide sits. This is in accordance with previous findings that the ${ }^{125} \mathrm{I}$-azidoglibenclamide but not ${ }^{125} \mathrm{I}$-glibenclamide can label Kir6.2 subunit (Aguilar-Bryan et al., 1990;
Schwanstecher et al., 1994b). Because the length of KNtp is conserved and limited (Fig. 2G), the special position of the Kir6.2 N-terminus intuitively suggests that it attenuates channel gating by acting as a chain to restrain the rotation of CTD, which is important for Kir family channel gating (Whorton and MacKinnon, 2011, 2013). To further validate this model, we inserted one to three Gly-Ser dipeptides (GS) between amino acid R27 and T28 of Kir6.2, a position before the structured Kir6.2 CTD and not conserved in the primary sequence (Fig. 2G), suggesting this position is not directly involved in SUR1 binding. We hypothesized that the KNtp of these mutants are still able to bind SUR1 but the restriction of the CTD rotation by KNtp might be reduced. Indeed, we found that the inhibitory effect of GBM progressively becomes weaker as the linker length increases (Fig. 2H). The SUR1Kir6.2 fusion construct demonstrated little GBM inhibition (Figs. $2 \mathrm{H}$ and $\mathrm{S} 1 \mathrm{~B}$ ), a phenotype similar to the $\mathrm{K}_{\mathrm{ATP}}$ channel assembled with KNtp deleted Kir6.2, suggesting the KNtp in the fusion construct cannot bind SUR1 anymore, because of the covalent linker. Moreover, without sulphonylureas, truncation of KNtp increases channel activity and reduces ATP sensitivity (Babenko et al., 1999; Koster, 1999; Reimann, 1999) and synthetic KNtp dose-dependently attenuates ATP inhibition (Babenko and Bryan, 2002), suggesting KNtp might remain bound to SUR1 when ATP, but no sulphonylurea, is present, albeit with decreased affinity. When we superposed our SUR1 ABC transporter module model of the GBM + ATP state to that from the another group (PDB: 6BAA) (Martin et al., 2017a), we found that there is a small but noticeable change of overall conformation of SUR 1 and the angle between M10 and $\mathrm{M} 16$ is $42.7^{\circ}$ in our structure whereas $39.3^{\circ}$ in their structure (Fig. S4F). Whether the observed difference of SUR1 conformation is due to KNtp binding discussed above or different data processing procedure still waits further investigation.

\section{Mg-independent ATP binding site on SUR1 NBD1}

During cryo-EM data processing, 3D classification demonstrated continuous conformational heterogeneity of SUR1 in the ATP + GBM state. Specifically, four peripheral SUR1 subunits wobble around the central Kir6.2 channel in small angles. This greatly limited the resolution of the overall cryoEM density map and deteriorated local map quality of the peripheral SUR1 ABC transporter module. Further improvement of SUR1 map quality at the ATP + GBM state benefited from focused refinement with local search on symmetry-expanded (Zhou et al., 2015) and signal-subtracted particles of the SUR1 ABC-transporter module (Bai et al., 2015). The map of "the isolated" SUR1 ABC-transporter module reached a resolution of $4.4 \AA$ and demonstrated markedly enhanced quality, especially for NBDs (Fig. S2C). The map also suggests a register shift of residues 1,061-1,079 on M13 in the structure by another group (PDB: 6BAA) (Martin et al., 2017a). More interestingly, we observed extra density on the degenerate site of SUR1 NBD1. The shape and size of this density matched that of 
ATPYS (Fig. 2I). Moreover, this density was at the expected location of nucleotides in the nucleotide-bound NBD structure of other ABC transporters (Zhang et al., 2017). Therefore, we modeled this density as an ATPYS molecule. We observed a similar density in the map of the ATP state structure (Fig. 1A). In the sample preparation procedure, we added $2 \mathrm{mmol} / \mathrm{L}$ ethylenediaminetetraacetic acid (EDTA) to avoid the activation effect of ATPYS by chelating divalent ions (Proks et al., 2010). Therefore, we observed an Mgindependent ATP binding site in the degenerate site on the NBD1 of SUR1. It is possible that in the ATP + GBM structure reported by another group (Martin et al., 2017a), ATP molecule is also bound on the NBD1, but limited local map quality (EMD-7073) precludes any conclusion.

\section{Architecture of the $\mathrm{K}_{\mathrm{ATP}}$ channel in the Mg-ADP bound state}

It is previously reported that KNtp is not necessary for channel opening and activation by Mg-ADP (Reimann, 1999). The fact that our 39AA fusion construct can be reactivated by Mg-ADP (Fig. S1B) suggests this construct can be used for structural study on the Mg-ADP activation mechanism. To trap SUR1 in an NBD-dimerized conformation, we supplemented the $\mathrm{K}_{\text {ATP }}$ fusion protein with Mg-ADP, the endogenous activator of the $\mathrm{K}_{\mathrm{ATP}}$ channel. We also added NN414, a SUR1 specific $\mathrm{KCO} ; \mathrm{VO}_{4}{ }^{3-}$, an ion that mimics the post-hydrolytic form of ATP $\mathrm{Y}$-phosphate; and 08:0 $\mathrm{PI}(4,5) \mathrm{P}_{2}$ lipid, a soluble $\mathrm{PIP}_{2}$ analogue which is required for $\mathrm{K}_{\mathrm{ATP}}$ channel activation. The structure of $\mathrm{K}_{\mathrm{ATP}}$ channel in the ADP bound state was solved to an overall resolution of $4.2 \AA$ (Fig. S5 and Table S3). We observed similar "T state" and "R state" conformational heterogeneity of the Kir6.2 CTD and SUR1 ABC transporter module as in the ATP + GBM state or the ATP state. The map quality of specific regions was dramatically improved by focused classification and refinement (Fig. S5 and Table S3).
The overall architecture of $\mathrm{K}_{\text {ATP }}$ in the ADP bound state is also similar to the ATP + GBM state or the ATP state (Fig. 3A and $3 B$ ). Four Kir6.2 channel subunits are located at the center, and four SUR1 subunits are in the periphery. The SUR1 ABC transporter module connects to the Kir6.2 via SUR1 TMD0-L0 fragment. This is in great contrast to the "quatrefoil form" structure of Mg-ATP and Mg-ADP (Lee et al., 2017) which has a disordered lasso motif and a large rotation of the SUR1 transporter module. The "quatrefoil form" might represent a "decoupled" state, in which the conformational change of SUR1 cannot be transferred to Kir6.2 by either the TMD0-L0 or the N-terminus of Kir6.2. Our overall structure is similar to the coupled "propeller form" (Lee et al., 2017), which has an ordered lasso motif, despite some positional shift of SUR1 subunits (Fig. S6A and S6B). The large difference between the "quatrefoil form" and the "propeller form" might arise from different sample preparation procedures, because detergents were replaced by amphipols in their experiments (Lee et al., 2017). Similarly to the ATP or ATP + GBM state, the CTD of Kir6.2 in "T state" has a clockwise rotation $\left(\sim 11.8^{\circ}\right)$ relative to that of "R state" (Fig. 3C). The two nucleotide binding sites on SUR1 are occupied by Mg-ADP, which results in an NBD-dimerized conformation (Fig. 3A and $3 \mathrm{~B}$ ). Moreover, the ATP binding sites of Kir6.2 are also occupied by ADP molecules, and the Kir6.2 channel is in the closed conformation, similarly to the ATP + GBM state (Figs. 3A, 3B and S6C), which is consistent with the result that ADP can inhibit the $\mathrm{K}_{\text {ATP }}$ channel albeit at a lower affinity (Hopkins et al., 1992; Schwanstecher et al., 1994a). The ADP binding mode at the inhibitory site of Kir6.2 is similar to that of ATP (Martin et al., 2017a) or ATPYS (Fig. S6C). Because ADP lacks the $y$ phosphate, there is no direct interaction between ADP and the $\mathrm{Y}$-phosphate-coordinating residue R50 (Martin et al., 2017b). This explains why ADP binds Kir6.2 with a lower affinity and the R50 mutation shifts the $\mathrm{IC}_{50}$ curve to a similar range of ADP $\beta S$ on wild-type channels (Schwanstecher et al., 1994a;
A

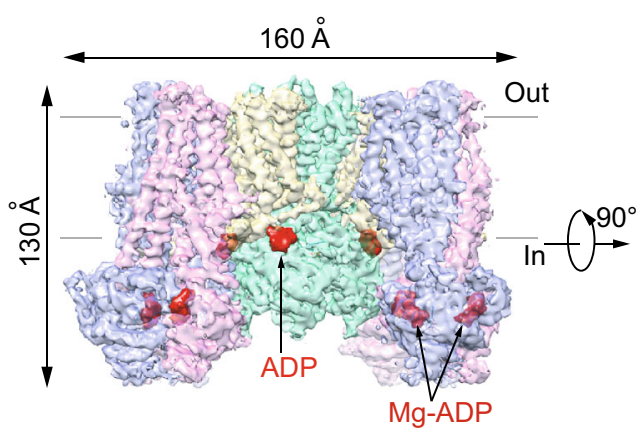

Mg-ADP state

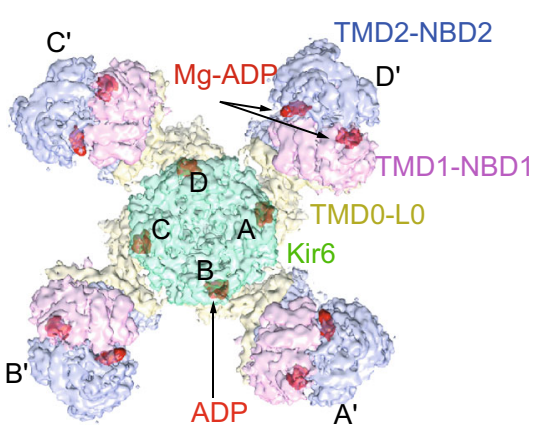

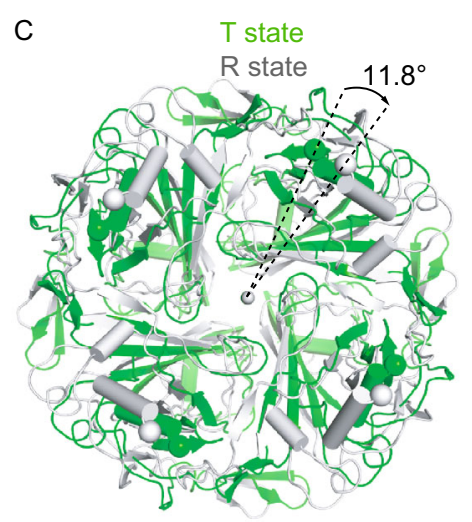

Figure 3. Structure of $K_{A T P}$ in the Mg-ADP state. (A) Side view of the $K_{A T P}$ structure in the Mg-ADP state, colored as in Fig. $1 A$. $A D P$ and Mg-ADP densities are colored in red. (B) Bottom view of the $K_{A T P}$ channel in the Mg-ADP state. (C) Bottom view of the aligned Kir6.2 structures between "T state" (green) and "R state" (gray) of the Mg-ADP state. Rotation angle between CTDs is measured similarly as in Fig. $1 \mathrm{E}$. 
Shimomura et al., 2006). We did not observe any density at the $\mathrm{PIP}_{2}$ binding site, probably due to the low affinity of the 08:0 $\mathrm{PI}(4,5) \mathrm{P}_{2}$ to the purified protein and also the negative cooperativity of inhibitory nucleotide binding and $\mathrm{PIP}_{2}$ binding on Kir6.2 (Baukrowitz et al., 1998; Hilgemann and Ball, 1996; Shyng and Nichols, 1998). This is in accordance with the fact that we observed a closed state Kir6.2 structure in the Mg-ADP state, because $\mathrm{PIP}_{2}$ is necessary for channel opening (Baukrowitz et al., 1998; Shyng and Nichols, 1998).

\section{Asymmetric NBD dimer of SUR1 in the Mg-ADP bound state}

In our focused refined map of SUR1 in the Mg-ADP state, we observed the densities of Mg-ADP molecules in both the degenerate and consensus sites (Fig. 4A-C). We did not observe densities of $\mathrm{VO}_{4}{ }^{3-}$, correlating with the fact that $\mathrm{VO}_{4}{ }^{3-}$ do not markedly affect SUR1 activity (Fig. S1B) (Proks, 1999; Shyng et al., 1997; Ueda et al., 1997). The Mg-ADP molecule in the degenerate site interacts with residues from both NBDs to induce a full closure of the degenerate site (Fig. 4C and 4D). In contrast, the Mg-ADP molecule in the consensus site primarily interacts with NBD2, and the consensus site is open (Fig. 4C and $4 \mathrm{E}$ ). We use the distance between the $\mathrm{C} \alpha$ atoms of the conserved glycine on the $A B C$ Walker A motif and the serine residue (a cysteine on SUR1 degenerate site) on the signature motif as an indicator of NBS closure. The distance between $\mathrm{Ca}$ of $\mathrm{G} 1485$ and $\mathrm{C} 717$ in the degenerate site is $11.5 \AA$, while the distance between $\mathrm{C} \alpha$ of $\mathrm{G} 833$ and $\mathrm{S} 1383$ in the consensus site is $13.0 \AA$ (Fig. 4F). These distances are the opposites of the NBD-dimerized state structure of CFTR with Mg-ATP bound (PDB: 5W81) (Zhang et al., 2017). For CFTR, the degenerate site is partially open, and the consensus site is fully closed. The distance between $\mathrm{G} 1350$ and S461 in the degenerate site is $12.7 \AA$, while the distance between $\mathrm{G} 550$ and $\mathrm{S} 1249$ in the consensus site is $10.6 \AA$ (Fig. 4G). The Mg-ADP state structure has the same asymmetric NBD-dimerized conformation as the Mg-ATP\&ADP state structure reported by another group (PDB: 6C3P EMD-7339) (Lee et al., 2017). In particular, Mg-ADP molecule induces similar full closure of degenerate site compared to the Mg-ATP molecules (Fig. S6D).

\section{Conformational changes of SUR1 ABC transporter module upon Mg-ADP binding}

By aligning the TMD0 domain of SUR1 between the ADP and ATP + GBM states, we found a large conformational change of the SUR1 ABC transporter module (Fig. 5A). The full closure of the degenerated site induced by Mg-ADP binding leads to the asymmetric dimerization of two NBDs (Fig. 5B). These conformational changes are further conveyed to the transmembrane domain via coupling helices that interact with NBDs to drive two halves of the transmembrane domain of SUR1 ABC transporter module to move closer (Fig. 5C). Specifically, M14 and M17 move toward M8 and M11 (Fig. 5C). As a result, SUR1 central cavity shrinks, and the GBM and KNtp sites are disrupted. We added NN414, a KCO, to our sample (Fig. S7A). We observed two positive densities in SUR1. One is close to the top of SUR1, surrounded by M10-11 from TMD1, and M12 and M17 from TMD2 (Fig. S7B). The other is at the central cavity surrounded by M8 from TMD1, and M15-17 from TMD2 (Fig. S7C). Both of the densities are absence in the Mg-ATP\&ADP state map, where NN414 was not added into the sample (EMD-7338, PDB: 6C3O) (Fig. S7B and S7C). By comparing the structures of transmembrane domain with (Mg-ADP state) and without NN414 (Mg-ATP\&ADP state), the ligand corresponding to density 1 introduces local structural changes of M9-11 (Fig. S7D). But whether these densities represent NN414 needs further studies. Notably, we did not observe any density that might account for KNtp. Both our Mg-ADP state structure reported here and the MgATP\&ADP state structure reported by another group (Lee et al., 2017) are captured using the fusion construct, where the authentic KNtp is not present. It is possible that the KNtp might adopt an unknown conformation that is different from the one observed in the ATP + GBM state but compatible with our current Mg-ADP state structure.

\section{Nucleotide binding induced NBD dimerization of SUR1}

In the ATP and ATP + GBM state structures, SUR1 is in an inward-facing conformation. We observed ATP density only in the NBD1 degenerate site but not in the NBD2 consensus site, which suggests that the degenerate site has a higher affinity for Mg-free ATP, and ATP alone is not able to drive the dimerization of SUR1 NBDs. This correlates with previous studies that NBD1 of SUR1 can bind 8-azido-ATP with high affinity in an Mg-independent manner (Matsuo et al., 1999a; Matsuo et al., 1999b; Ueda et al., 1997). In addition, the subsequent binding of ATP to NBD1 can be strongly antagonized by prebound Mg-ADP (Ueda et al., 1997), indicating that the degenerate site of NBD1 can be occupied by Mg-ADP as well. Indeed, in our Mg-ADP state structure, we observed Mg-ADP molecules in both the NBD1 degenerate site and the NBD2 consensus site.

The asymmetric NBD dimer conformation observed in the Mg-ADP state structure suggests that the Mg-ADP molecule in the closed degenerate site directly mediates the formation of the NBD dimer interface, because this Mg-ADP molecule physically interacts with both NBDs. In contrast, the Mg-ADP molecule in the open consensus site interacts only with NBD2 but not with NBD1, indicating that it does not contribute directly to NBD dimer formation. By comparing the structures of individual NBD monomer in different states, we found a noticeable conformational change in NBD2 but not in NBD1 (Fig. 5D). Induced conformational changes of the NBD by nucleotide binding were previously observed in the crystal structures of isolated NBD of $A B C$ transporter MJ1267 (Karpowich et al., 2001) and in the cryo-EM structure of CFTR (Zhang and Chen, 2016; Zhang et al., 2017) 
A

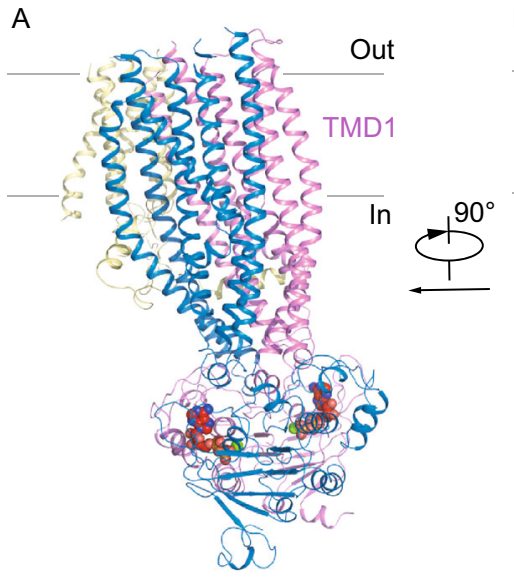

B

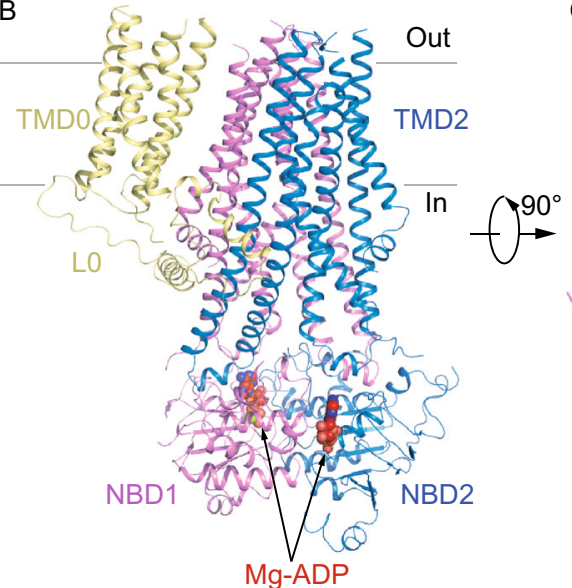

C

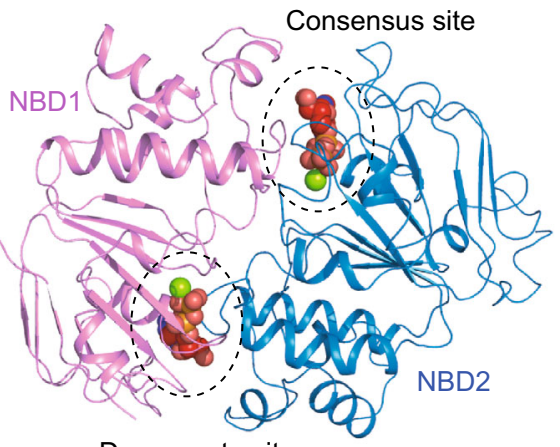

Degenerate site
D

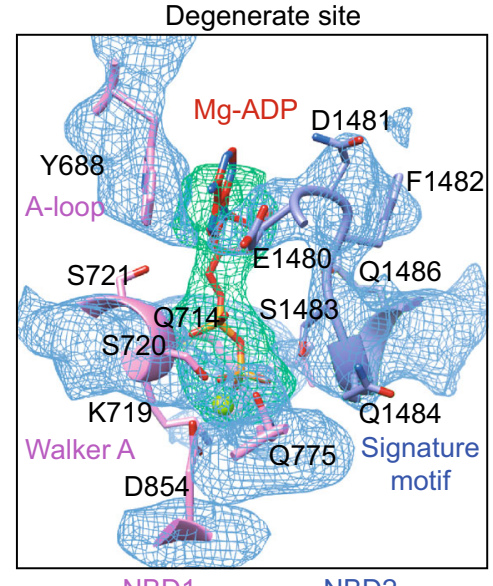

NBD1

F

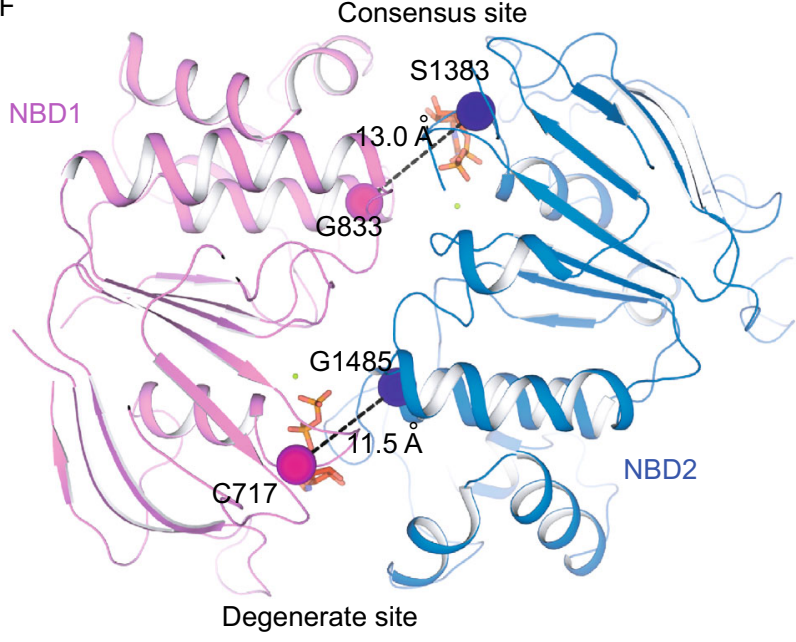

SUR1
E

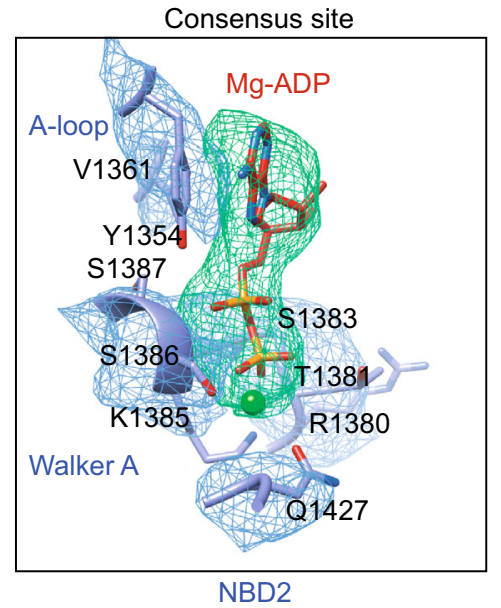

G

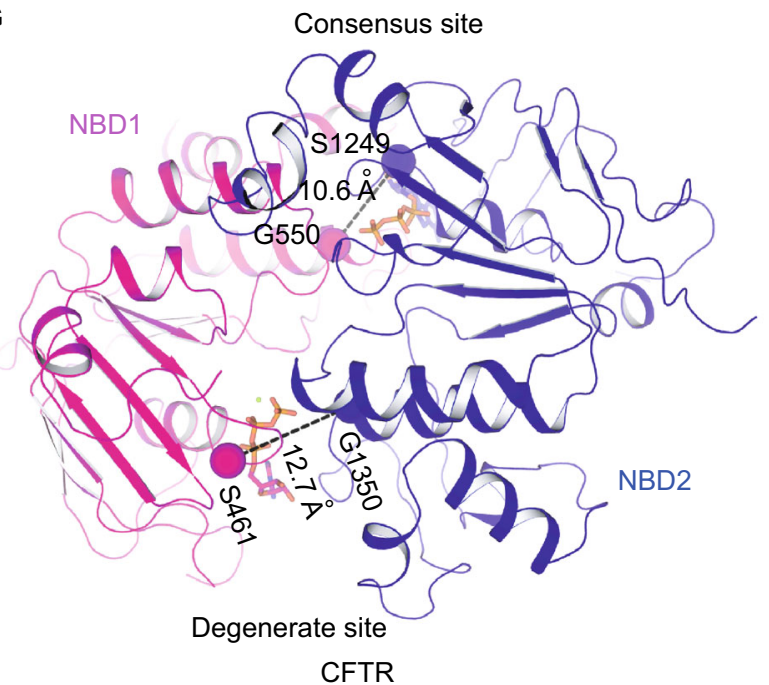

Figure 4. Mg-ADP on SUR1. (A) Side view of a single SUR1 subunit in cartoon representation. Color scheme is the same as in Fig. $1 \mathrm{~A} . \mathrm{Mg}^{2+}$ and ADP are shown as green and red spheres, respectively. (B) Side view of a single SUR1 subunit with a $90^{\circ}$ rotation relative to panel (A). (C) Bottom view of a single SUR1 subunit with a $90^{\circ}$ rotation relative to (B). For simplicity, only NBD1 and NBD2 are shown. Two Mg-ADP molecules are circled. (D and E) EM densities at the degenerate site (D) and consensus site (E), colored as in Fig. $2 \mathrm{H} . \mathrm{Mg}^{2+}$ is shown as a green sphere. ( $F$ and G) Differences of NBD closures in SUR1 (F) and CFTR (PDB: 5W81) (G). C $\alpha$ distances between the glycine in the Walker A motif and serine (cysteine instead at the degenerate site of SUR1) in the ABC signature motif are shown as dashed lines. 
A

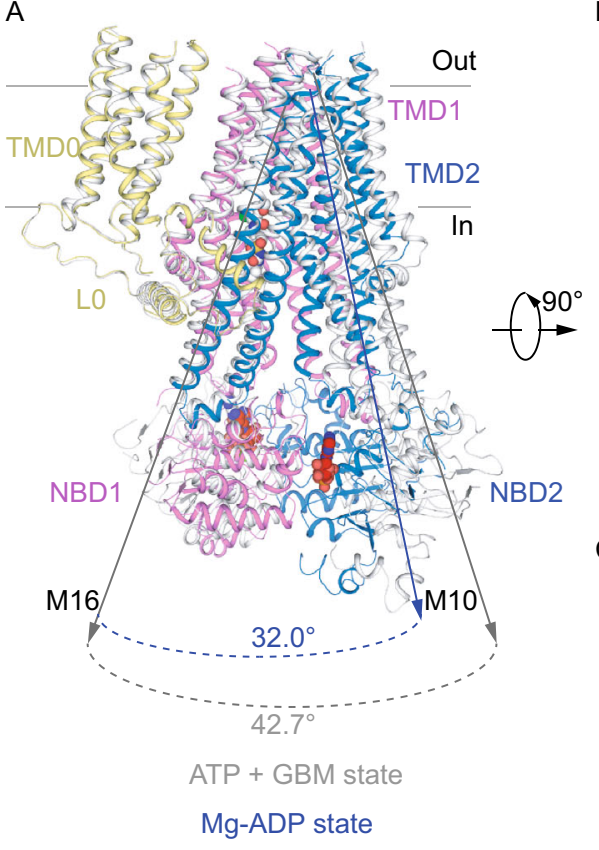
B

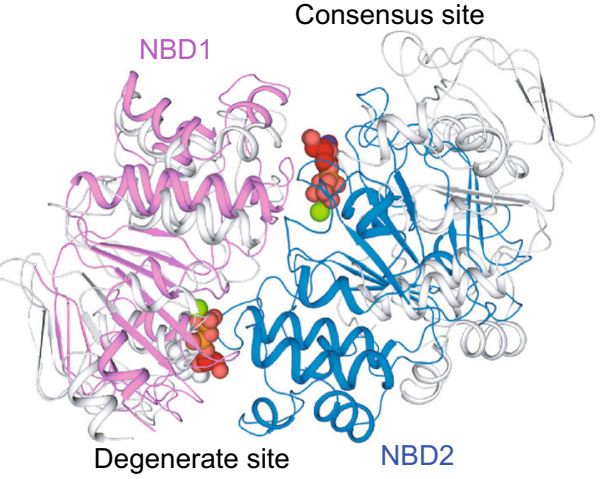

C

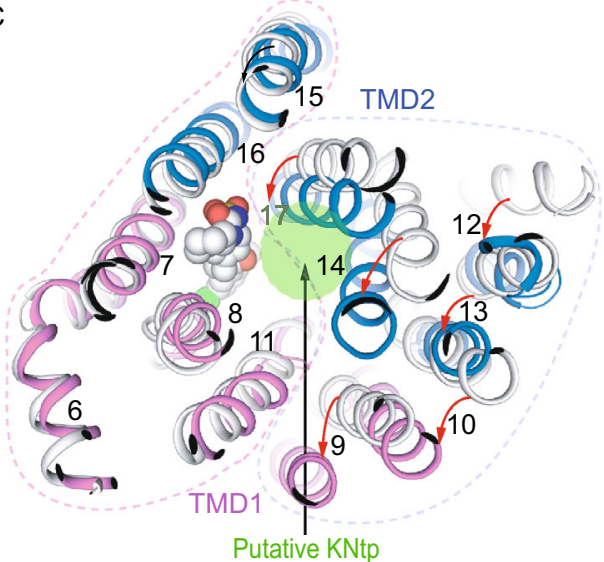

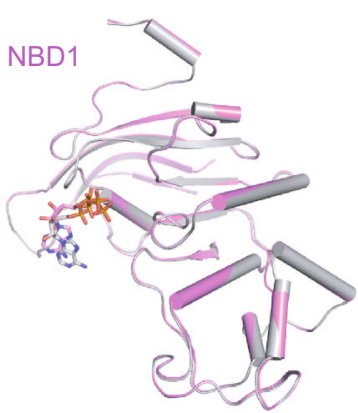

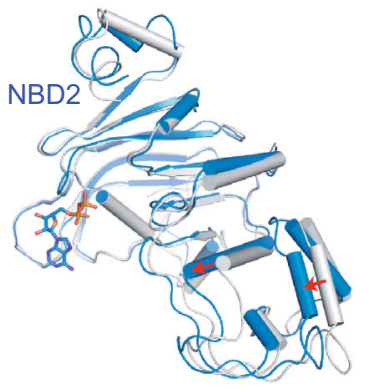

Figure 5. Conformational changes of SUR1. (A) Superposition of the Mg-ADP bound structure (colored) and the ATP + GBM bound structure (gray) by aligning the TMD0 domain of SUR1. Angles between helices M10 and M16 in the two structures are labeled. Mg-ADP, ATPYS and GBM molecules are all shown as spheres. (B) Bottom view of NBDs of the $\mathrm{K}_{\text {ATP }}$ channel with a $90^{\circ}$ rotation relative to $(A)$. (C) Bottom view of TMDs of the $K_{A T P}$ channel with a $90^{\circ}$ rotation relative to (A). Positional changes of transmembrane helices from the first half of TMDs (M9-10, 12-14 and 17) are indicated by red arrows. The dashed lines specify the two halves of TMDs. The putative KNtp binding site is shown as a green circle, showing the displacement of GBM-binding sites upon nucleotide binding. (D) Structural comparisons of individual NBD1 (top) or NBD2 (bottom) between the ATP + GBM state (gray) and the Mg-ADP state (colored). Conformational changes of $\alpha$ helices-rich subdomain in NBD2 are indicated by red arrows. NBD structures are aligned according to $\beta$ sheet-rich subdomain.

and also were sampled in MD-simulations (Jones and George, 2017). The conformational changes of NBD2 observed here might partially be due to the Mg-ADP binding and full closure of the degenerate site, but we suggest that the Mg-ADP binding on the NBD2 consensus site plays a more prominent role. This is supported by the previous radioactive 8-azido-ATP photo cross-linking results. First, the NBD2 consensus site mutation K1385M greatly reduces the ability of prebound Mg-ADP to prevent further ATP binding on NBD1, when Mg-ADP and ATP were added to SUR1 sequentially (Ueda et al., 1997; Ueda et al., 1999), suggesting that Mg-ADP acts on the NBD2 consensus site to allosterically increase the affinity of Mg-ADP on the NBD1 degenerate site. Second, Mg-ADP or Mg-ATP binding on the NBD2 consensus site can stabilize prebound ATP on the NBD1 degenerate site (Ueda et al., 1999), indicating that nucleotide binding on the NBD2 consensus site can send a signal to the degenerate site to increase its affinity for nucleotides, probably via the conformational change of NBD2, as we observed here.

\section{Model for SUR1 conformational change}

On the basis of experiments and structures of $\mathrm{K}_{\mathrm{ATP}}$ channels in complex with different ligand combinations solved with different constructs, we propose the following hypothetic model for the conformational change of SUR1 subunit (Fig. 6A-H). When GBM is bound, GBM and the Kir6.2 $\mathrm{N}$-terminus cooperatively bind inside SUR1 to inhibit channel gating (Fig. 6A and 6E). Without GBM, the Kir6.2 N-terminus can still bind to the central cavity of SUR1 to inhibit channel gating but with decreased affinity. In the presence of ATP, ATP can occupy the NBD1 degenerate site, but the NBDs are still separated (Fig. 6B and 6F). When the ADP 
A
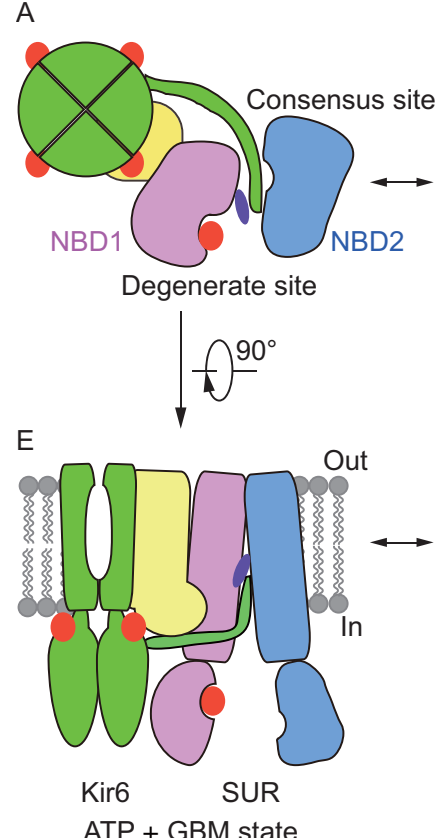

$\mathrm{F}$

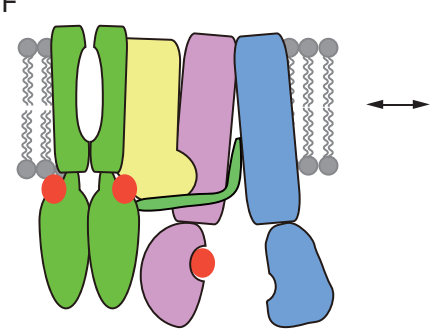

ATP state

G

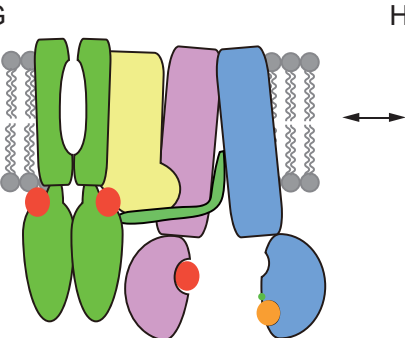

Intermediate state
C

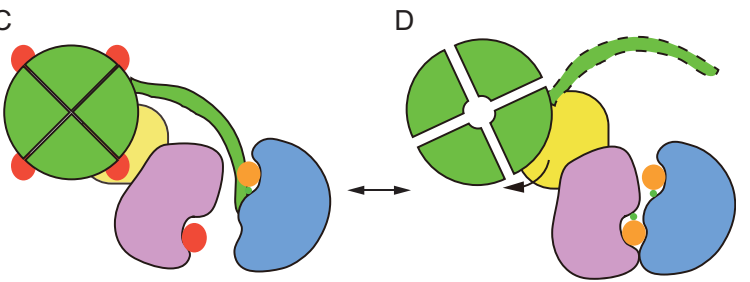

$\mathrm{D}$

Figure 6. Model for nucleotide-induced NBD dimerization of the $\mathrm{K}_{\text {ATP }}$ channel. (A-D) Bottom view of SUR1 cartoon models from cytosolic side. For simplicity, only CTDs of Kir6.2 and TMD0-L0 and NBDs from one SUR1 subunit are shown and colored the same as in Fig. 1A. KNtp with an unknown conformation in Mg-ADP state is outlined by dashed lines. ATP and ADP are shown as red and orange big spheres, respectively. $\mathrm{Mg}^{2+}$ ions are shown as green small spheres. $(\mathrm{E}-\mathrm{H})$ Side view of SUR1 cartoon models. For simplicity, a pair of Kir6.2 and one SUR1 subunit are shown.

concentration increases, Mg-ADP first binds to the NBD2 consensus site to induce a conformational change of NBD2 and enhance the affinity of the NBD1 degenerate site for MgADP or Mg-ATP (Fig. 6C and 6G). Then, prebound ATP together with $\mathrm{Mg}$ ion or newly bound Mg-ADP bridges two halves of the degenerate site to induce its full closure and NBD dimerization. Finally, there is a global conformational change of SUR1, which allosterically activates the Kir6.2 channel and occludes the GBM site of SUR1, and Kir6.2 $\mathrm{N}$-terminus relocates accordingly (Fig. 6D and 6H). Mg-ATP binding at the NBD2 consensus site can have similar functions as Mg-ADP, albeit with lower affinity (Vedovato et al., 2015). Our model suggests that the essential elements for asymmetric NBD dimerization are the degenerate site of both NBDs and the consensus site of NBD2. Indeed, previous mutation results support this model. Mutations of K719A and $\mathrm{D} 854 \mathrm{~N}$ on the degenerate site of NBD1; G1479D, G1479R, G1485D, G1485R and Q1486H on the degenerate site of NBD2; and K1385M, D1506N, and D1506A on the consensus site of NBD2 (Gribble et al., 1997; Nichols et al., 1996; Shyng et al., 1997) can impair Mg-ADP activation, whereas mutations of G827D, G827R, and Q834H on the consensus site of NBD1 can preserve Mg-ADP activation, although with altered kinetics (Nichols et al., 1996; Shyng et al., 1997). Our model also suggests that ATP hydrolysis is not required for channel activation, which is consistent with functional studies (Choi et al., 2008; Ortiz et al., 2013).

\section{MATERIALS AND METHODS}

\section{Cell culture}

Sf9 cells (Thermo Fisher Scientific) were cultured in Sf-900 III SFM medium (Thermo Fisher Scientific) at $27{ }^{\circ} \mathrm{C}$. FreeStyle 293-F (Thermo Fisher Scientific) suspension cells were cultured in Freestyle 293 medium (Thermo Fisher Scientific) supplemented with $1 \%$ FBS at $37{ }^{\circ} \mathrm{C}$ with $6 \% \mathrm{CO}_{2}$ and $70 \%$ humidity.

Screening of fusion constructs

Fusion constructs, SUR1-Kir6.2, were created by linking the C-terminus of SUR1 from Mesocricetus auratus (Aguilar-Bryan et al., 1995) to the N-terminus of Kir6.2 (Woo et al., 2013) from Mus musculus using flexible linkers with variable lengths. The fusion constructs were cloned into a modified C terminal GFP-tagged BacMam expression vector which contains two Strep tags after GFP as described previously (Li et al., 2017). SUR1-Kir6.2 fusion constructs were screened by transient transfection of FreeStyle 293-F cells (Thermo Fisher Scientific) cultured in SMM-293TI medium (Sino Biological Inc.) using polyethylenimine (Polyscience). Cells were harvested $60 \mathrm{~h}$ posttransfection and solubilized in $20 \mathrm{mmol} / \mathrm{L}$ HEPES pH8.0 + $120 \mathrm{mmol} / \mathrm{L}$ $\mathrm{KCl}+1 \%$ digitonin (Biosynth) at $25^{\circ} \mathrm{C}$. Cell lysates were cleared by centrifuge at $40,000 \mathrm{rpm}$ for $30 \mathrm{~min}$ and supernatants were loaded onto Superose 6 increase column (GE Healthcare) for analysis (Kawate and Gouaux, 2006). Based on expression level and SUR1Kir6.2 tetramer assembly, SUR1-Kir6.2 fusion with a 39-residue linker (VDGSGSGSGSAAGSGSGSGSGSGAAGSGSGSGSGSGAAA) 
was selected and expressed in BacMam system for further structural characterization (Goehring et al., 2014).

\section{Electrophysiology}

$\mathrm{K}_{\text {ATP }}$ constructs were transfected into FreeStyle 293-F cells at density of $1 \times 10^{6}$ cells $/ \mathrm{mL}$ using polyethylenimine and cells were cultured $36-48 \mathrm{~h}$ before recording. Patch electrodes were pulled from a horizontal microelectrode puller ( $P-1000$, Sutter Instrument Co, USA) to a tip resistance of 3.0-5.0 M . The pipette solution contained (mmol/L): $140 \mathrm{KCl}, 1.2 \mathrm{MgCl}_{2}, 2.6 \mathrm{CaCl}_{2}, 10 \mathrm{HEPES}(\mathrm{pH}$ $7.4, \mathrm{NaOH})$ and the bath solution contained (mmol/L): $140 \mathrm{KCl}, 10$ EGTA, $1 \mathrm{MgCl}_{2}, 10$ HEPES ( $\mathrm{pH} 7.4, \mathrm{NaOH}$ ). Macroscopic currents were recorded using inside-out mode at $+60 \mathrm{mV}$ through an Axopatch 200B amplifier (Axon Instruments, USA). Recording were started when currents were stable after patch excision. Signals were acquired at $20 \mathrm{kHz}$ and lowpass filtered at $5 \mathrm{kHz}$. Data was further analyzed with pclampfit 9.0 software and filtered at $1 \mathrm{kHz}$. For the GBM inhibition assay, steady state currents after GBM treatment were normalized to the currents before GBM treatment. Run-down was not corrected during data analysis.

\section{Protein expression and purification}

$\mathrm{K}_{\text {ATP }}$ channels were expressed in FreeStyle 293-F cells as described previously ( $\mathrm{Li}$ et al., 2017). For purification, membrane pellets were homogenized in $20 \mathrm{mmol} / \mathrm{L}$ HEPES pH 8.0 at $25^{\circ} \mathrm{C}+120$ $\mathrm{mmol} / \mathrm{L} \mathrm{KCl}$ and then solubilized in $1 \%$ digitonin for $30 \mathrm{~min}$ at $4{ }^{\circ} \mathrm{C}$. Unsolubilized materials were removed by centrifugation at 40,000 rpm for 50 min in Ti45 rotor (Beckman). Supernatant was loaded onto $5 \mathrm{~mL}$ strep-tactin superflow high capacity resin (IBA). Resin was washed by $20 \mathrm{mmol} / \mathrm{L}$ HEPES pH 8.0 at $25^{\circ} \mathrm{C}+120 \mathrm{mmol} / \mathrm{L}$ $\mathrm{KCl}+0.1 \%$ digitonin and protein was eluted by $20 \mathrm{mmol} / \mathrm{L}$ HEPES $\mathrm{pH} 8.0$ at $25^{\circ} \mathrm{C}+120 \mathrm{mmol} / \mathrm{L} \mathrm{KCl}+0.1 \%$ digitonin $+1 \mathrm{mmol} / \mathrm{L}$ EDTA $+5 \mathrm{mmol} / \mathrm{L}$ desthiobiotin. Protein eluted from strep-tactin column was concentrated by $100-k D a$ cut-off concentrator (Millipore) and loaded onto Superose 6 increase (GE Healthcare) running in 20 $\mathrm{mmol} / \mathrm{L}$ HEPES pH 8.0 at $25^{\circ} \mathrm{C}+120 \mathrm{mmol} / \mathrm{L} \mathrm{KCl}+0.1 \%$ digitonin. Peak fractions that contain SUR1-Kir6.2 fusion protein were combined and concentrated to $A_{280}=8$ (estimated protein concentration was $9 \mu \mathrm{mol} / \mathrm{L} \mathrm{K}_{\text {ATP }}$ proteins). For cryo-EM sample preparation, the proteins were supplemented with $2 \mathrm{mmol} / \mathrm{L}$ EDTA, $200 \mu \mathrm{mol} / \mathrm{L}$ glibenclamide (TCl), and $2 \mathrm{mmol} / \mathrm{L}$ ATPYS (Sigma) (ATP + GBM state), or $2 \mathrm{mmol} / \mathrm{L}$ EDTA and $2 \mathrm{mmol} / \mathrm{L}$ ATPYS (Sigma) (ATP state), or $2.5 \mathrm{mmol} / \mathrm{L} \mathrm{MgCl}_{2}, 1 \mathrm{mmol} / \mathrm{L}$ ADP (Sigma), $400 \mu \mathrm{mol} / \mathrm{L} \mathrm{08:0} \mathrm{PI}$ $(4,5) \mathrm{P}_{2}$ (Avanti), $500 \mu \mathrm{mol} / \mathrm{L} \mathrm{NN414}$ (Sigma), and $1 \mathrm{mmol} / \mathrm{L} \mathrm{Na}_{3} \mathrm{VO}_{4}$ (Mg-ADP state). Cryo-EM sample preparation was prepared as described previously (Li et al., 2017).

\section{Cryo-EM data acquisition}

Cryo-grids were screened on a Talos (Thermo Fisher Scientific) operated at a voltage of $200 \mathrm{kV}$ with an eagle $4 \mathrm{k} \times 4 \mathrm{k} C \mathrm{CD}$ camera (Thermo Fisher Scientific). Good grids were transferred into a Titan Krios (Thermo Fisher Scientific) operated at $300 \mathrm{kV}$ and images were collected using K2 camera (Gatan) mounted post a quantum energy filter with $20 \mathrm{eV}$ slit. K2 camera was operated under super resolution mode with super resolution pixel size $0.5275 \AA$ (ATP +
GBM state and Mg-ADP state) or $0.67 \AA$ (ATP state) at object plane. The defocus value ranged from $-1.0 \mu \mathrm{m}$ to $-3.5 \mu \mathrm{m}$. Data acquisitions were performed automatically using Leginon software (Suloway et al., 2005) in the movie mode with a dose rate of 3.8 or 4.7 $\mathrm{e}^{-} / \mathrm{s} / \AA^{2}$. The total exposure was $50 \mathrm{e}^{-} / \AA^{2}$ and each 13 or 11 seconds movie was dose-fractioned into 50 frames.

\section{Image processing}

Collected movies were gain-corrected, motion-corrected, exposurefiltered and binned with MotionCor2 (Zheng et al., 2017), producing dose-weighted and summed micrographs with pixel size $1.055 \AA /$ pixel (ATP + GBM state and Mg-ADP state) or $1.34 \AA$ A/pixel (ATP state). CTF models of dose-weighted micrographs were determined using Gctf (Zhang, 2016). Autopick was done with Gautomatch (developed by Kai Zhang, MRC-LMB) using the projections of our previous map (EMD-6689) as template. Auto-picked particles were extracted from dose-weighted micrographs by binning factor of 2 and subjected to $2 \mathrm{D}$ classification using cryoSPARC (Punjani et al., 2017). 3D classification was done using our former $\mathrm{K}_{\text {ATP }}$ map (EMD-6689) as the initial model with GPU-accelerated Relion 2.0 (Kimanius et al., 2016). Particles from selected 3D classes were re-centered and re-extracted from summed micrographs without binning (1.055 $\AA /$ pixel or $1.34 \AA /$ pixel). Re-extracted particles were subjected to $3 D$ auto-refinement with C4 symmetry. Additional focused classification of Kir6.2 CTD with a soft mask but without alignment was used to separate 3D classes with different CTD conformations. To improve the local map quality of SUR1 ABC transporter module (maSUR1 214-1579), alignment parameters of each SUR1 ABC transporter module particles were obtained from global refined particles by symmetry expansion using relion_particle_symmetry_expand-sym C4 (Zhou et al., 2015). Then the signals outside SUR1 ABC transporter module were subtracted from each particles using particle subtraction function in Relion 2.0 as described previously (Bai et al., 2015). The signal subtracted particles were used for further $3 \mathrm{D}$ refinement using local search by restricting sigma_ang to $5^{\circ}$, using the map of SUR 1 ABC transporter module as the starting model. All of the resolution estimations were based on gold standard FSC 0.143 after correction for mask effects (Chen et al., 2013). The final map was sharpened with B factor automatically determined by Relion 2.0 (Kimanius et al., 2016).

\section{Model building}

Individual domain of previous $\mathrm{K}_{\text {ATP }}$ models (PDB: 5WUA and 5TWV) and CFTR in Mg-ATP bound state (PDB: 5W81) were used as the initial model, docked into cryo-EM map with best local quality of that region by chimera and manually rebuilt in Coot according to the density (Emsley et al., 2010). The model was further refined by phenix (Adams et al., 2010). Specifically, models of Kir6.2 transmembrane domain and SUR1 TMD0 were refined against the focused refined map of transmembrane domain in the ATP + GBM state. Models of SUR1 ABC transporter module were refined against the focused refined map in respective states. Models of Kir6.2 CTD were refined against maps after focused classification and refinement of Kir6.2 CTD in the ATP + GBM state. The individually refined models were then docked into the maps of the full $\mathrm{K}_{\text {ATP }}$ channel and refined respectively. Figures were prepared with Pymol (Schrödinger, LLC.) or Chimera (Pettersen et al., 2004). 
Quantification and statistical analysis

Resolution estimations of cryo-EM density maps are based on the 0.143 Fourier Shell Correlation (FSC) criterion (Chen et al., 2013).

\section{ACCESSION CODES}

The 3D cryo-EM density maps have been deposited in the Electron Microscopy Data Bank under the accession number EMD: EMD6831, EMD-6832, and EMD-6833 for the ATP + GBM state of $\mathrm{K}_{\text {ATP }}$, EMD-6848, EMD-6849, and EMD-6850 for the ATP state of $K_{\text {ATP, }}$ and EMD-6852, EMD-6851, and EMD-6853 for the Mg-ADP state of $\mathrm{K}_{\text {ATP. }}$ Coordinates of the $\mathrm{K}_{\text {ATP }}$ structure have been deposited in the Protein Data Bank under the accession number PDB: 5YKE, 5YKF, $5 Y K G, 5 Y W 7$ for the ATP + GBM state of $K_{A T P}, 5 Y W 8,5 Y W 9$, and 5YWA for the ATP state of $\mathrm{K}_{\mathrm{ATP}}$, and $5 \mathrm{YWC}, 5 \mathrm{YWB}$, and 5YWD for the Mg-ADP state of $\mathrm{K}_{\mathrm{ATP}}$.

\section{ACKNOWLEDGMENTS}

We thank all of Chen Lab members for kindly help. We also thank Joseph Bryan for sharing maSUR1 cDNA and J. Marc Simard for sharing mmKir6.2 cDNA. Cryo-EM data collection was supported by electron microscopy laboratory and Cryo-EM platform of Peking University with the assistance of Xuemei $\mathrm{Li}$ and Daqi $\mathrm{Yu}$, the National Center for Protein Science (Shanghai) with assistance of Liangliang Kong and Zhenglin Fu, Center for Biological Imaging, Institute of Biophysics, Chinese Academy of Sciences with assistance of Zhenxi Guo. Part of structural computation was also performed on the Computing Platform of the Center for Life Science and High-performance Computing Platform of Peking University.

The work is supported by grants from the Ministry of Science and Technology of China (National Key R\&D Program of China, 2016YFA0502004 to Lei Chen) and National Natural Science Foundation of China (Grant Nos. 31622021 and 31521062 to Lei Chen) and Young Thousand Talents Program of China to Lei Chen and the China Postdoctoral Science Foundation (2016M600856 and 2017 T100014 to Jing-Xiang Wu). Jing-Xiang Wu is supported by the postdoctoral foundation of the Peking-Tsinghua Center for Life Sciences, Peking University.

\section{ABBREVIATIONS}

ABC, ATP-binding cassettes; cryo-EM, cryo-electron microscopy; GBM, glibenclamide; $K_{\text {ATP, }}$ ATP-sensitive potassium channel; Kir6, inward-rectifying potassium channel 6 ; NBD, nucleotide binding domain; SUR, sulfonylurea receptor; TMDO-LO, transmembrane domain 0-loop 0

\section{COMPLIANCE WITH ETHICS GUIDELINES}

Jing-Xiang Wu, Dian Ding, Mengmeng Wang, Yunlu Kang, Xin Zeng and Lei Chen declare that they have no conflict of interest. This article does not contain any studies with human or animal subjects performed by the any of the authors.

\section{AUTHOR CONTRIBUTIONS}

Lei Chen initiated the project. Jiang-Xiang Wu, Dian Ding, Mengmeng Wang, Yunlu Kang, Xin Zeng and Lei Chen designed experiments. Jiang-Xiang $\mathrm{Wu}$ prepared the cryo-EM sample. Jiang-Xiang $\mathrm{Wu}$, Mengmeng Wang, Dian Ding, Yunlu Kang and Lei Chen collected EM data. Jiang-Xiang $\mathrm{Wu}$ and Lei Chen performed image processing and analyzed EM data. Dian Ding performed electrophysiology experiments. Xin Zeng performed the HPLC and mass spectrum analysis. Lei Chen and Jiang-Xiang Wu built the model, prepared the figures and wrote the manuscript draft. All authors contributed to manuscript preparation.

\section{OPEN ACCESS}

This article is distributed under the terms of the Creative Commons Attribution 4.0 International License (http://creativecommons.org/ licenses/by/4.0/), which permits unrestricted use, distribution, and reproduction in any medium, provided you give appropriate credit to the original author(s) and the source, provide a link to the Creative Commons license, and indicate if changes were made.

\section{REFERENCES}

Adams PD et al (2010) PHENIX: a comprehensive Python-based system for macromolecular structure solution. Acta Crystallogr D Biol Crystallogr 66:213-221

Aguilar-Bryan L et al (1990) Photoaffinity labeling and partial purification of the beta cell sulfonylurea receptor using a novel, biologically active glyburide analog. J Biol Chem 265:8218-8224

Aguilar-Bryan L et al (1995) Cloning of the beta cell high-affinity sulfonylurea receptor: a regulator of insulin secretion. Science 268:423-426

Aittoniemi $J$ et al (2009) Review. SUR1: a unique ATP-binding cassette protein that functions as an ion channel regulator. Philos Trans R Soc Lond B Biol Sci 364:257-267

Ashcroft FM et al (2017) Neonatal diabetes and the KATP channel: from mutation to therapy. Trends Endocrinol Metab 28:377-387

Babenko AP, Bryan J (2002) SUR-dependent modulation of KATP channels by an N-terminal KIR6.2 peptide. Defining intersubunit gating interactions. J Biol Chem 277:43997-44004

Babenko AP et al (1999) The N-terminus of KIR6.2 limits spontaneous bursting and modulates the ATP-inhibition of KATP channels. Biochem Biophys Res Commun 255:231-238

Bai XC et al (2015) Sampling the conformational space of the catalytic subunit of human gamma-secretase. Elife. https://doi. org/10.7554/eLife.11182

Baukrowitz T et al (1998) PIP2 and PIP as determinants for ATP inhibition of KATP channels. Science 282:1141-1144

Bryan J et al (2005) Insulin secretagogues, sulfonylurea receptors and K(ATP) channels. Curr Pharm Des 11:2699-2716

Carr RD et al (2003) NN414, a SUR1/Kir6.2-selective potassium channel opener, reduces blood glucose and improves glucose tolerance in the VDF Zucker rat. Diabetes 52:2513-2518 
Chen S et al (2013) High-resolution noise substitution to measure overfitting and validate resolution in $3 \mathrm{D}$ structure determination by single particle electron cryomicroscopy. Ultramicroscopy 135:24-35

Choi KH et al (2008) Testing for violations of microscopic reversibility in ATP-sensitive potassium channel gating. J Phys Chem B 112:10314-10321

Clement JPT et al (1997) Association and stoichiometry of K(ATP) channel subunits. Neuron 18:827-838

Devaraneni PK et al (2015) Structurally distinct ligands rescue biogenesis defects of the KATP channel complex via a converging mechanism. J Biol Chem 290:7980-7991

Emsley $P$ et al (2010) Features and development of coot. Acta Crystallogr D Biol Crystallogr 66:486-501

Flagg TP et al (2010) Muscle KATP channels: recent insights to energy sensing and myoprotection. Physiol Rev 90:799-829

Goehring A et al (2014) Screening and large-scale expression of membrane proteins in mammalian cells for structural studies. Nat Protoc 9:2574-2585

Gribble FM et al (1997) The interaction of nucleotides with the tolbutamide block of cloned ATP-sensitive $\mathrm{K}^{+}$channel currents expressed in Xenopus oocytes: a reinterpretation. J Physiol 504 (Pt 1):35-45

Hibino $\mathrm{H}$ et al (2010) Inwardly rectifying potassium channels: their structure, function, and physiological roles. Physiol Rev 90:291366

Hilgemann DW, Ball R (1996) Regulation of cardiac $\mathrm{Na}^{+}, \mathrm{Ca}^{2+}$ exchange and KATP potassium channels by PIP2. Science 273:956-959

Hopkins WF et al (1992) Two sites for adenine-nucleotide regulation of ATP-sensitive potassium channels in mouse pancreatic betacells and HIT cells. J Membr Biol 129:287-295

Jones PM, George AM (2017) How intrinsic dynamics mediates the allosteric mechanism in the $A B C$ transporter nucleotide binding domain dimer. J Chem Theory Comput 13:1712-1722

Karpowich $\mathrm{N}$ et al (2001) Crystal structures of the MJ1267 ATP binding cassette reveal an induced-fit effect at the ATPase active site of an ABC transporter. Structure 9:571-586

Kawate T, Gouaux E (2006) Fluorescence-detection size-exclusion chromatography for precrystallization screening of integral membrane proteins. Structure 14:673-681

Kimanius D et al (2016) Accelerated cryo-EM structure determination with parallelisation using GPUs in RELION-2. Elife. https:// doi.org/10.7554/eLife.18722

Koster JC et al (1999) ATP inhibition of KATP channels: control of nucleotide sensitivity by the $\mathrm{N}$-terminal domain of the Kir6.2 subunit. J Physiol 515(Pt 1):19-30

Kuhner $\mathrm{P}$ et al (2012) Importance of the Kir6.2 N-terminus for the interaction of glibenclamide and repaglinide with the pancreatic $\mathrm{K}$ (ATP) channel. Naunyn Schmiedebergs Arch Pharmacol 385:299-311

Lee KPK et al (2017) Molecular structure of human KATP in complex with ATP and ADP. Elife. https://doi.org/10.7554/eLife.32481

Li N et al (2017) Structure of a pancreatic ATP-sensitive potassium channel. Cell 168:101-110

Locher KP (2016) Mechanistic diversity in ATP-binding cassette $(A B C)$ transporters. Nat Struct Mol Biol 23:487-493
Martin GM et al (2017a) Anti-diabetic drug binding site in a mammalian KATP channel revealed by Cryo-EM. Elife. https:// doi.org/10.7554/eLife.31054

Martin GM et al (2017b) Cryo-EM structure of the ATP-sensitive potassium channel illuminates mechanisms of assembly and gating. Elife. https://doi.org/10.7554/eLife.24149

Matsuo $\mathrm{M}$ et al (1999a) ATP binding properties of the nucleotidebinding folds of SUR1. J Biol Chem 274:37479-37482

Matsuo $M$ et al (1999b) NEM modification prevents high-affinity ATP binding to the first nucleotide binding fold of the sulphonylurea receptor, SUR1. FEBS Lett 458:292-294

Nichols CG et al (1996) Adenosine diphosphate as an intracellular regulator of insulin secretion. Science 272:1785-1787

Ortiz D et al (2013) Reinterpreting the action of ATP analogs on $\mathrm{K}$ (ATP) channels. J Biol Chem 288:18894-18902

Pettersen EF et al (2004) UCSF Chimera-a visualization system for exploratory research and analysis. J Comput Chem 25:16051612

Proks $P$ et al (1999) Involvement of the N-terminus of Kir6.2 in the inhibition of the KATP channel by ATP. J Physiol 514(Pt 1):19-25

Proks $P$ et al (2010) Activation of the K(ATP) channel by Mgnucleotide interaction with SUR1. J Gen Physiol 136:389-405

Punjani A et al (2017) cryoSPARC: algorithms for rapid unsupervised cryo-EM structure determination. Nat Methods 14:290-296

Reimann $F$ et al (1999) Involvement of the n-terminus of Kir6.2 in coupling to the sulphonylurea receptor. J Physiol 518(Pt 2):325336

Schwanstecher C et al (1994a) Interaction of tolbutamide and cytosolic nucleotides in controlling the ATP-sensitive $\mathrm{K}^{+}$channel in mouse beta-cells. Br J Pharmacol 111:302-310

Schwanstecher M et al (1994b) Identification of a 38-kDa high affinity sulfonylurea-binding peptide in insulin-secreting cells and cerebral cortex. J Biol Chem 269:17768-17771

Shimomura K et al (2006) Mutations at the same residue (R50) of Kir6.2 (KCNJ11) that cause neonatal diabetes produce different functional effects. Diabetes 55:1705-1712

Shyng S, Nichols CG (1997) Octameric stoichiometry of the KATP channel complex. J Gen Physiol 110:655-664

Shyng SL, Nichols CG (1998) Membrane phospholipid control of nucleotide sensitivity of KATP channels. Science 282:1138-1141

Shyng $S$ et al (1997) Regulation of KATP channel activity by diazoxide and MgADP. Distinct functions of the two nucleotide binding folds of the sulfonylurea receptor. J Gen Physiol 110:643-654

Suloway C et al (2005) Automated molecular microscopy: the new Leginon system. J Struct Biol 151:41-60

Ueda $\mathrm{K}$ et al (1997) MgADP antagonism to $\mathrm{Mg}^{2+}$-independent ATP binding of the sulfonylurea receptor SUR1. J Biol Chem 272:22983-22986

Ueda K et al (1999) Cooperative binding of ATP and MgADP in the sulfonylurea receptor is modulated by glibenclamide. Proc Natl Acad Sci USA 96:1268-1272

Vedovato $\mathrm{N}$ et al (2015) The nucleotide-binding sites of SUR1: a mechanistic model. Biophys J 109:2452-2460

Vila-Carriles WH et al (2007) Defining a binding pocket for sulfonylureas in ATP-sensitive potassium channels. FASEB $\mathrm{J}$ 21:18-25 
Whorton MR, MacKinnon R (2011) Crystal structure of the mammalian GIRK2 $\mathrm{K}^{+}$channel and gating regulation by $\mathrm{G}$ proteins, PIP2, and sodium. Cell 147:199-208

Whorton MR, MacKinnon R (2013) X-ray structure of the mammalian GIRK2-betagamma G-protein complex. Nature 498:190-197

Woo SK et al (2013) The sulfonylurea receptor 1 (Sur1)-transient receptor potential melastatin 4 (Trpm4) channel. J Biol Chem 288:3655-3667

Zhang K (2016) Gctf: real-time CTF determination and correction. J Struct Biol 193:1-12

Zhang Z, Chen J (2016) Atomic structure of the cystic fibrosis transmembrane conductance regulator. Cell 167(1586-1597):e1589
Zhang $Z$ et al (2017) Conformational changes of CFTR upon phosphorylation and ATP binding. Cell 170(483-491):e488

Zhao $Y$ et al (2015) In vitro inhibition of AKR1Cs by sulphonylureas and the structural basis. Chem Biol Interact 240:310-315

Zheng SQ et al (2017) MotionCor2: anisotropic correction of beaminduced motion for improved cryo-electron microscopy. Nat Methods 14:331-332

Zhou M et al (2015) Atomic structure of the apoptosome: mechanism of cytochrome c- and dATP-mediated activation of Apaf-1. Genes Dev 29:2349-2361 